\title{
Nonequilibrium phase transition in a model for the propagation of innovations among economic agents
}

\author{
Mateu Llas, ${ }^{1}$ Pablo M. Gleiser, ${ }^{1}$ Juan M. López, ${ }^{2}$ and Albert Díaz-Guilera ${ }^{1}$ \\ ${ }^{1}$ Departament de Física Fonamental, Universitat de Barcelona, Avenida Diagonal 647, E-08028 Barcelona, Spain \\ ${ }^{2}$ Instituto de Física de Cantabria (CSIC-UC), E-39005 Santander, Spain
}

(Received 30 July 2003; published 15 December 2003)

\begin{abstract}
We characterize the different morphological phases that occur in a simple one-dimensional model of propagation of innovations among economic agents [X. Guardiola et al., Phys. Rev E 66, 026121 (2002)]. We show that the model can be regarded as a nonequilibrium surface growth model. This allows us to demonstrate the presence of a continuous roughening transition between a flat (system size independent fluctuations) and a rough phase (system size dependent fluctuations). Finite-size scaling studies at the transition strongly suggest that the dynamic critical transition does not belong to directed percolation and, in fact, critical exponents do not seem to fit in any of the known universality classes of nonequilibrium phase transitions. Finally, we present an explanation for the occurrence of the roughening transition and argue that avalanche driven dynamics is responsible for the novel critical behavior.
\end{abstract}

DOI: 10.1103/PhysRevE.68.066101

PACS number(s): 89.65.-s, 89.75.Da, 05.45.-a, 68.35.Rh

\section{INTRODUCTION}

In the past few years there has been an increasing interest among theoretical physicists in complex phenomena occurring in fields that are far apart from the traditional realm of physics like social and economic sciences [1-4]. The main reason being that social and economic systems often exhibit many instances of complex dynamics, including selforganization, pattern formation, synchronization, and phase transitionlike phenomena that closely resemble those observed in nonequilibrium physical systems [5-8]. Physicists approach to these systems usually provides insights into the basic ingredients that should be included in simple models in order to obtain the dynamics observed. Although it is clear that physics inspired models of socioeconomic phenomena are often very simplistic views of very complicated systems, the aim is to show how complex macroscopic dynamics might arise from rather simple rules operating at the "microscopic" level of individual agents and their mutual interactions.

In this paper we consider a very simple model of innovation propagation dynamics in an economic system formed by agents $[9,10]$. The aim is to describe in a simple way the adoption of innovations that occur among industries, firms, or individuals. Once a brand new product appears in the market, the agents should decide whether or not they will incorporate the new technology. Adopting the new technology (in the form of a software, device, gadget, etc.) has a cost, but at the same time it may improve business performance in the case of firms, or may leveloff life quality for individuals. Innovations are regarded here in a broad sense and stand for any device or tool. For instance, a firm can decide to incorporate world wide web (WWW) technology by creating or revamping its WWW page, or going into e-commerce for the first time. A layman observation is that, if not always, in most cases, when the new technology actually improves performance its use will spread all over.

In this approach two main mechanisms for the propaga- tion of innovations are considered. First, external pressure can push an agent to adopt an innovation. This mechanism intends to mimic exogenous influence, such as advertising, and is independent of the network structure. Second, there is interaction among agents, which depends on the underlying network structure and is introduced in the model by considering local coupling rules. A single tunable parameter $C$, which is fixed and the same for all agents, accounts for the agents' resistance to change, and controls the dynamical behavior of the system. In earlier studies, some of us have already focused on the several outcomes of the model in the social and economic context $[9,11,12]$. From the economic point of view, the main result is that the system presents an optimal behavior for an intermediate value of $C$, and that this can be quantified with a macroscopic observable. This feature is closely related with the statistical properties of the profile of technological levels of the agents and its dynamical evolution. A proper characterization of these properties can be done with the tools of statistical mechanics and it is the main aim of this work.

In this paper we show that this model can be interpreted as a surface growth model. Such interpretation allows us to analyze the dynamical behavior of the model as a kinetic roughening process akin to other nonequilibrium surface growth systems. We find that the model exhibits a continuous phase transition between a rough and a flat phase at a critical value $C_{t h}$ of the control parameter. We focus on the scaling properties at the threshold in order to determine the critical exponents at the transition. By defining a convenient order parameter and studying its finite-size scaling properties near criticality we are able to show that the horizontal correlation length diverges as $\xi \sim\left|C-C_{t h}\right|^{-\nu}$, where $\nu \approx 2.5$. Close to the threshold, relaxation dynamics to the stationary regime is characterized by diverging correlation times $\tau \sim \xi^{z}$, where $z$ $\approx 0.57$ is the dynamic exponent. The existence of a nonequilibrium roughening transition in a $1+1$ dimension model makes it interesting also for statistical mechanics. It is known that phase transitions in nonequilibrium $1+1$ dimen- 


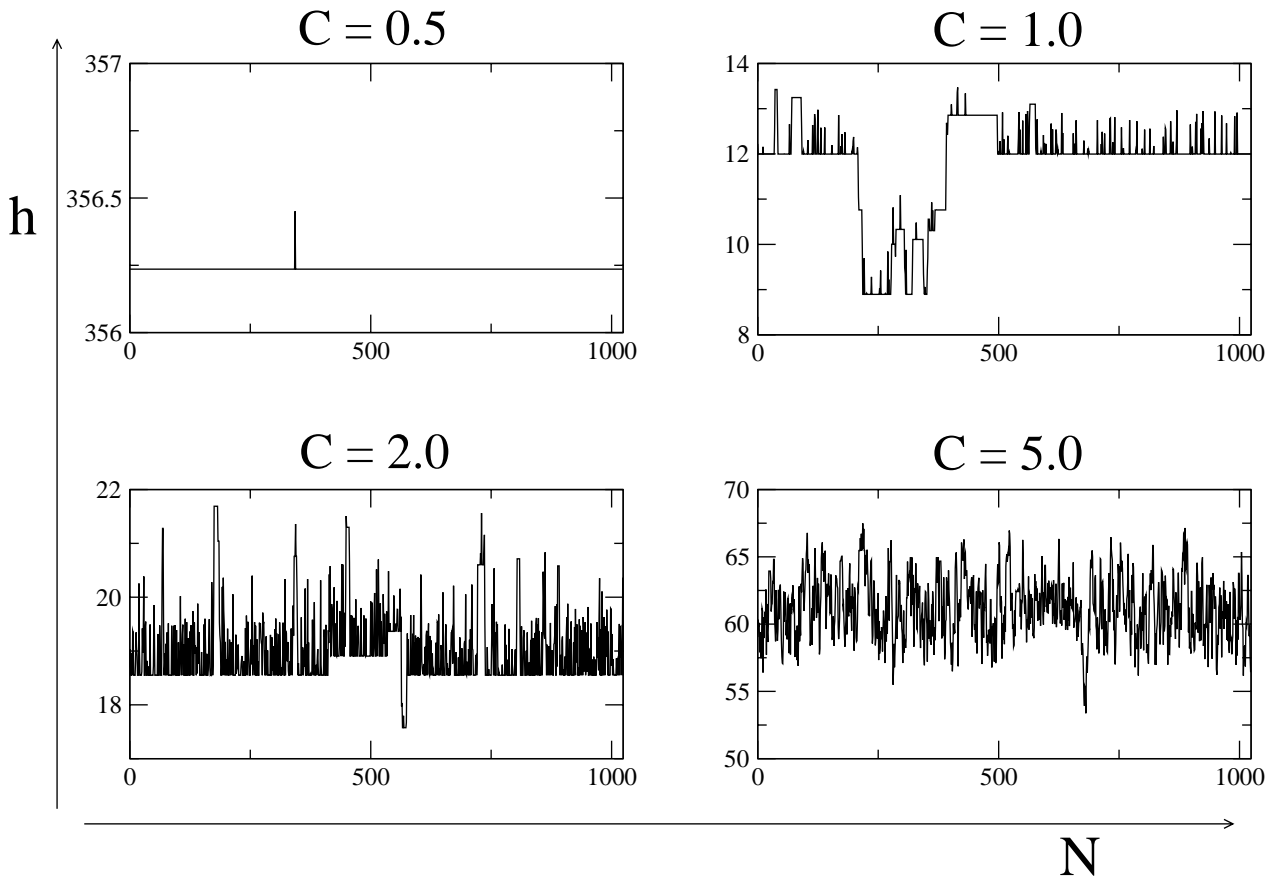

FIG. 1. Snapshots of the profile of a system with $N=1024$ for $C=0.5, C=1.0, C=2.0$, and $C=5.0$.

sional systems are usually associated with systems with absorbing states [13]. In this case, the number of absorbing states and symmetries among them determine the universality class to which a particular system belongs to. Thus, it is of great interest to find models far from equilibrium which do not possess absorbing states but still display a phase transition. As we will see below, our model lacks absorbing states and the measured critical exponents suggest that this model belongs to a different universality class. Finally, we discuss the physical mechanisms behind the critical transition in this model.

\section{THE MODEL}

We consider $N$ agents placed at the sites of a onedimensional lattice with periodic boundary conditions. Each site (agent) $i$ is characterized by a real variable $h_{i}$. In general, we can consider this quantity as a characteristic of a given individual that other agents might want to imitate. When an agent has adopted a new feature (innovation), her neighbors become aware of the change and balance their interest (quantified as $h_{i}-h_{j}$ ) with their resistance to change $C$ to decide if they would like to imitate this change. In this way $C$ controls the mechanism of imitation. This parameter is constant and the same for all the agents in the system.

The system is updated as follows [11]:

(1) At each time step an agent $h_{i}$ is randomly selected and

$$
h_{i} \rightarrow h_{i}+\Delta
$$

where $\Delta$ is a random variable uniformly distributed in $[0,1]$ [23]. The driving process accounts for the external pressure that may lead an individual to spontaneously update by adopting a new technology. This mechanism keeps the system out of equilibrium.

(2) The agents $j \in \Gamma(i), \Gamma(i)$ being the set of nearest neighbors of agent $i$, upgrade if $h_{i}-h_{j} \geqslant C$. If the latter is satisfied, agent $j$ imitates agent $i$ by setting $h_{j}=h_{i}$. In this way the information of an update may spread beyond the neighbors of the originally perturbed site. This procedure is repeated until no one else wants to change, concluding an avalanche of imitation events. We thus assume that the time scale of the imitation process is much shorter than the one corresponding to the external driving.

Starting from a flat initial condition, $h_{i}=0$ for all $i$, the system evolves to a stationary regime. In Fig. 1 we present snapshots of the surface profile in the stationary regime for four different values of $C$.

The time scale separation-namely, slow driving versus fast relaxation in the form of avalanches of activity-is similar to that occurring in self-organized critical (SOC) systems and dynamically drives the system towards a stationary state [14]. We will see below that, at variance with most SOC systems, two different stable phases are possible: an ordered (flat) phase and a disordered (rough) phase with scale invariant properties. For small $C$, the driving process easily triggers avalanches that cover the whole system, leading to a uniform advance and a flat phase. On the contrary, for large $C$, there are almost no avalanches, and the system advances mostly due to the random updates, thus presenting an extremely heterogeneous and rough profile. For intermediate values of $C$ one can clearly see the presence of large avalanches and new updates. In fact, in the intermediate regime one can find the optimal growth regime in which the agents reach a given average level with a minimum number of upgrades [9]. 

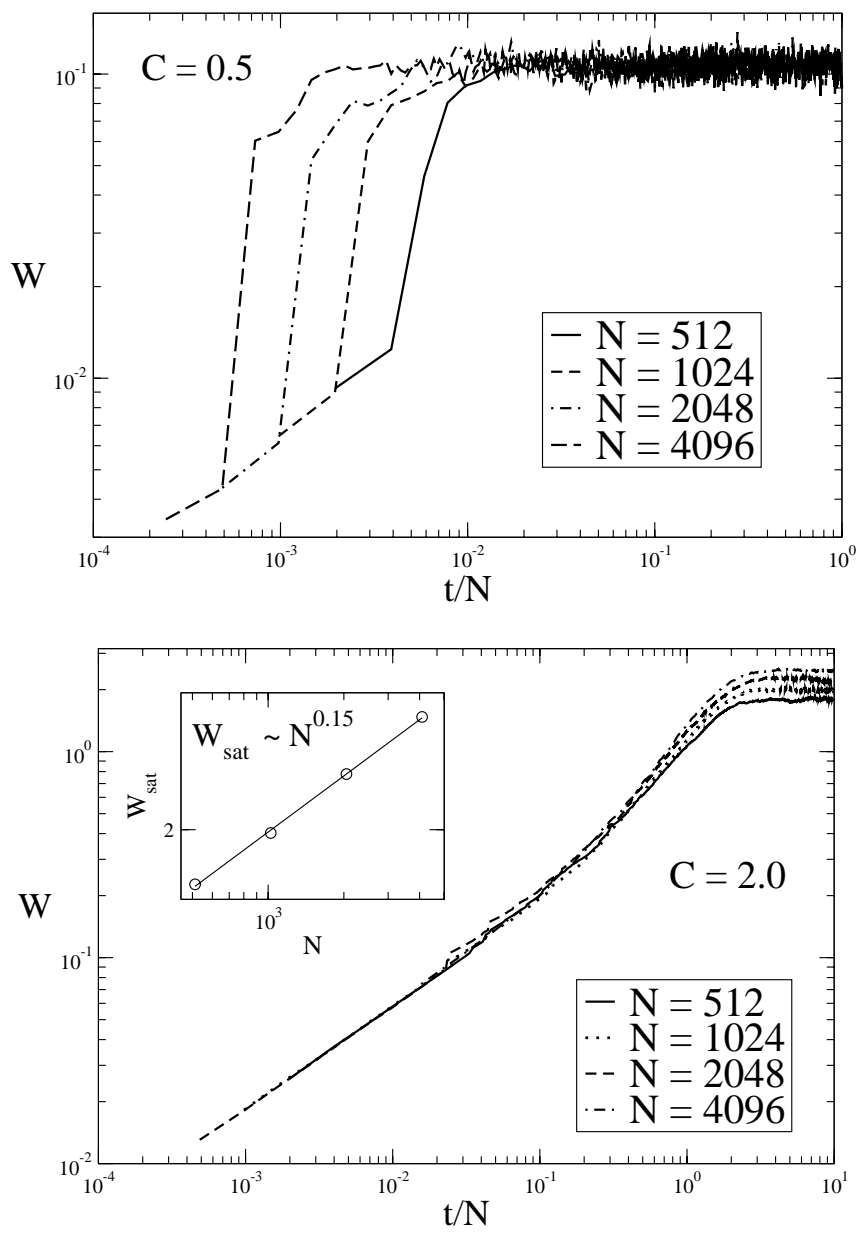

FIG. 2. Time evolution of the width $W(t)$ for four different system sizes $(N=512, N=1024, N=2048$, and $N=4096)$ when $C=0.5$ and $C=2.0$. Results correspond to averages over 500 realizations of the noise. The inset shows that $W_{\text {sat }}$ scales with system size.

\section{CRITICAL ROUGHENING TRANSITION}

\section{A. Stationary regime}

In order to characterize the different morphological phases we performed extensive numerical simulations of the model. The fluctuations of the profile height are measured by means of the global interface width [15],

$$
W(N, t)=\left\langle\sqrt{(1 / N) \sum_{i=1}^{N}\left[h_{i}(t)-\bar{h}(t)\right]^{2}}\right\rangle
$$

where \langle\rangle stands for average over noise realizations. At each time step the mean height value

$$
\bar{h}(t)=(1 / N) \sum_{i=1}^{N} h_{i}(t)
$$

is also calculated. It is important to stress here that time is always measured in the external driving temporal scale, so that one time step $t$ corresponds to an external update. As a consequence, the number of agents that change their state

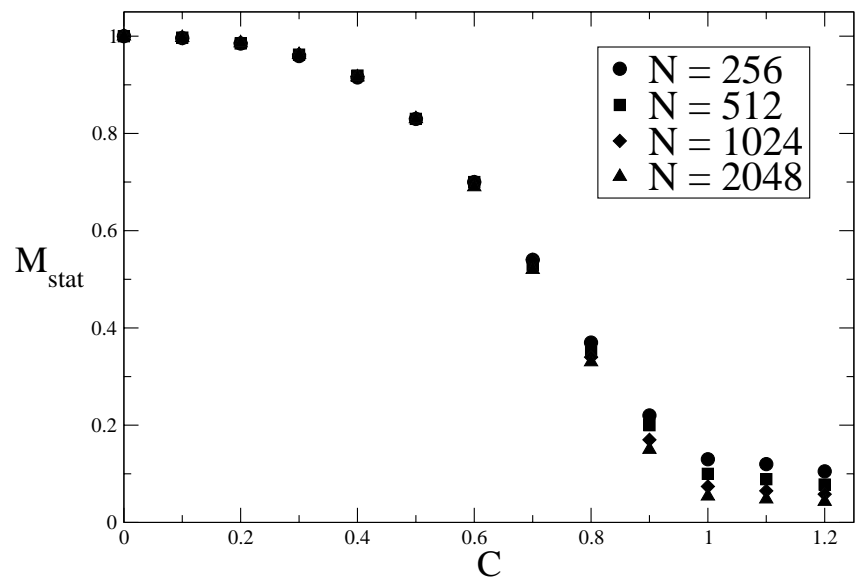

FIG. 3. Order parameter $M_{\text {stat }}$ vs $C$ for four different system sizes $(N=256, N=512, N=1024$, and $N=2048)$. The points correspond to an average over 250 realizations of the noise.

may vary from a single one (which changes from $h$ to $h$ $+\Delta)$ to any number of agents in the system if the update generates an avalanche.

In the following we report on the behavior of the width in the two different phases. On the top panel of Fig. 2 we show the behavior of $W(N, t)$ for $C=0.5$. The saturation value does not depend on the system size, which indicates that the system is in the smooth phase. On the bottom panel of Fig. 2 we show the numerical results in the rough phase for $C$ $=2.0$. In this case the saturation value $W_{\text {sat }}(N)$ scales with the system size, as is shown in the inset. We find that in the rough phase the height fluctuations seem to fit reasonably well with a scaling as $W_{\text {sat }}(N) \sim N^{0.15}$, which actually cannot be distinguished from a possibly logarithmic dependence. These results strongly suggest the presence of a roughening transition.

In order to study the critical behavior at the transition threshold we introduce a convenient order parameter. When looking at the profile snapshots in the stationary regime in Fig. 1, one can easily notice the presence of large plateaux, i.e., finite connected regions of agents that have the same height. The size of these flat regions decreases as $C$ grows, since for $C \rightarrow \infty$ the model has to become equivalent to the random deposition model [15]. We found that the size of the largest plateau can be used as an order parameter. In the following, we shall call $M$ to the size of the largest plateau in the system, normalized by the system size $N$. In this way, a completely flat profile corresponds to $M=1$. We have also tried other common choices, as the often used Ising-like magnetization $(1 / N) \sum_{i}(-1)^{h_{i}(t)}$ and its variations [16,17]. However, we found that our election has better scaling properties for this particular case, since it takes into account the singular behavior of the flat phase in this model.

Starting from a flat initial condition $M(t)$ evolves until it reaches a stationary value. In Fig. 3 we show the behavior of the stationary value of the order parameter $M_{\text {stat }}(N, C)$ vs $C$ for four different system sizes. The order parameter allows us to distinguish the two phases discussed above. Note that for small values of the control parameter, the system gets ordered, implying a flat phase. On the contrary, the stationary 


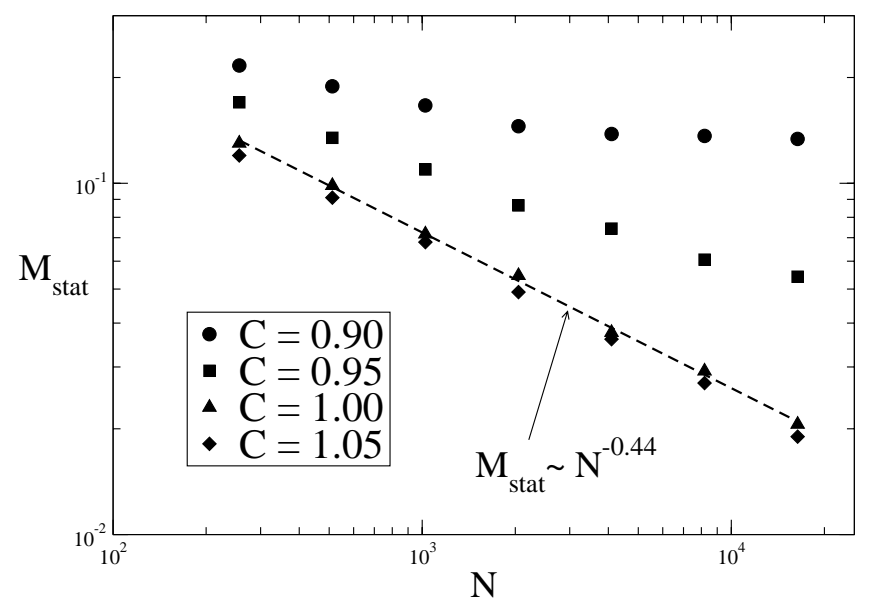

FIG. 4. Order parameter $M_{\text {stat }}$ vs $N$ for four different values of the parameter $C=0.90, C=0.95, C=1.00$, and $C=1.05$. A power law-decay $M_{\text {stat }} \sim N^{-0.44}$ is observed for $C=1.0$. Results correspond to averages over 500 realizations.

value of the order parameter goes to zero for large values of $C$ as the system size becomes larger. Critical behavior is expected close to the threshold $C_{t h}$ and, as usual, it can be studied numerically by finite-size scaling techniques [16-18] as follows. For any value of the control parameter $C$, there exists a horizontal correlation length $\xi$, which diverges as $\xi \sim \epsilon^{-\nu}$ when the distance to the critical threshold goes to zero $\epsilon=\left|C-C_{t h}\right| \rightarrow 0$. In finite systems this actually occurs for values of $C$ close to, but not exactly at, the threshold since the finite-size critical behavior is encountered as long as $\xi \sim N$, or equivalently when $\epsilon \sim N^{-1 / \nu}$. Close to the threshold, $\epsilon \rightarrow 0$, for sufficiently large values of the system size, $M_{\text {stat }}$ converges to a finite value obeying $M_{\text {stat }}(N, \epsilon)$ $\sim \epsilon^{\beta}$. Just at the critical point $\epsilon=0$ we expect the order parameter to decay as a power law with the system size

$$
M_{\text {stat }}(N, \epsilon=0) \sim N^{-\beta / \nu} .
$$

In Fig. 4 we plot our numerical results for $M_{\text {stat }}(N, \epsilon)$ vs $N$ for different values of the distance to the threshold $\epsilon$. Only for $C=C_{t h}$ a power law with the system size can be obtained and the slope of the straight line in a log-log plot gives an estimation of the ratio $\beta / \nu=0.44 \pm 0.05$ between critical exponents. We can thus identify $C_{t h}=1.0 \pm 0.1$ with the critical point. After having determined the critical point, numerical data for different system sizes can be cast in the finite-size scaling ansatz

$$
M_{\text {stat }}(N, \epsilon)=N^{-\beta / \nu} g\left(\epsilon N^{1 / \nu}\right)
$$

where the scaling function $g(y) \sim$ const for $y \ll 1$, and $g(y)$ $\sim y^{\beta}$ if $y \gg 1$. In Fig. 5 we plot a data collapse that allows us to determine the values of the exponents $1 / \nu=0.40 \pm 0.05$ and $\beta / \nu=0.44 \pm 0.05$. From these, we then have $\beta \sim 1.10$ and $\nu \sim 2.50$.

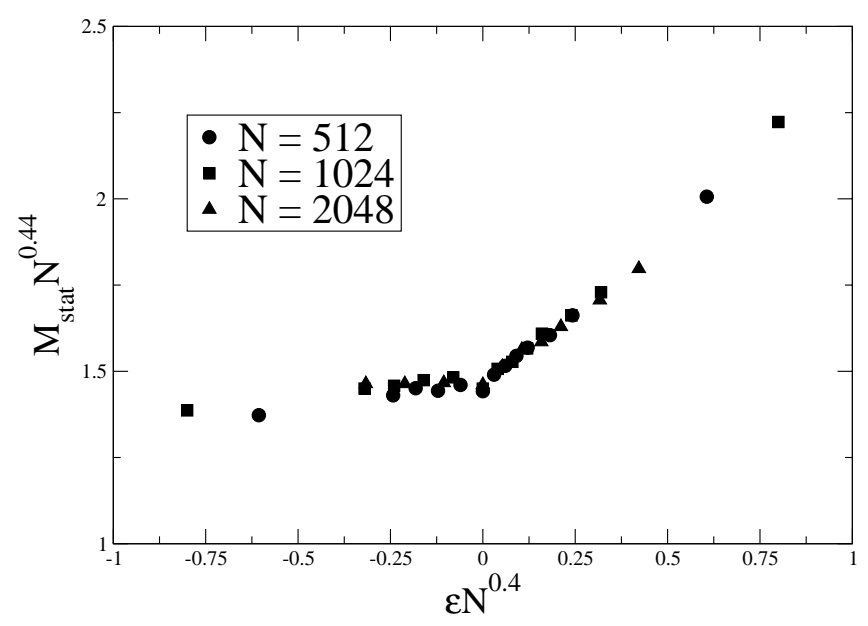

FIG. 5. Data collapse of the order parameter $M_{\text {stat }}$ as given in Eq. (5). Results correspond to averages over 500 realizations.

\section{B. Dynamics}

Since the model is out of equilibrium our study is completed next with an analysis of the dynamic behavior, which contains much information about the universality of the roughening transition. In Fig. 6 we show the temporal behavior of the order parameter $M(t, N, C)$ for three different values of $C$ (above, below, and at the critical threshold). Again, only at the critical point we may expect to find a power-law decay $M(t, N, \epsilon=0) \sim t^{-\beta / \nu_{t}}$, where $\nu_{t}$ is the exponent associated with the diverging correlation time $\tau \sim \epsilon^{-\nu_{t}}$ as $\epsilon \rightarrow 0$. The correlation time corresponds to the typical time that correlations survive in the system and is given by $\tau \sim \xi^{z}$, where $z$ is the dynamic exponent. The three exponents are related by the usual scaling relation $z=\nu_{t} / \nu$ provided dynamic scaling holds. In Fig. 6 we can see that only at the critical point

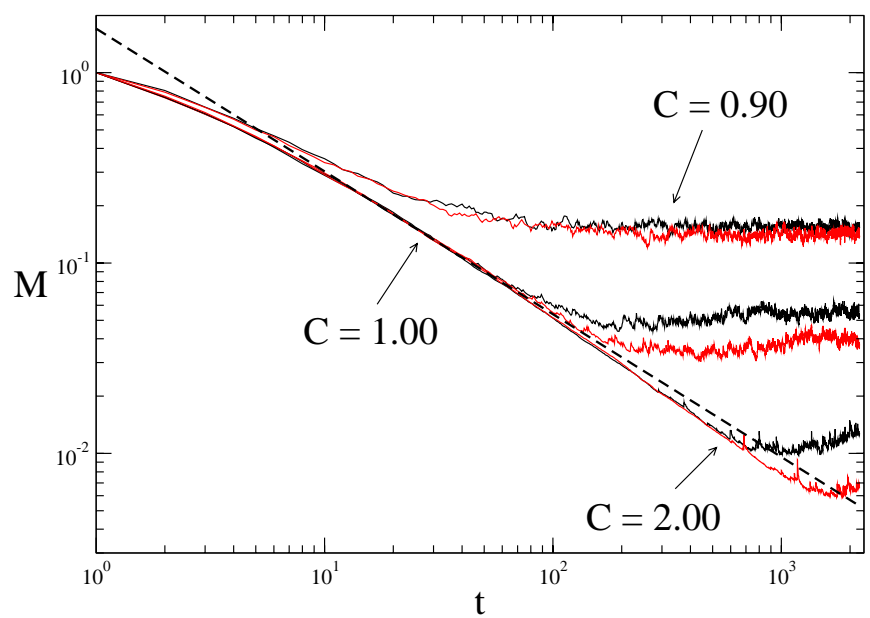

FIG. 6. Order parameter dynamics for three values of $C$ : $C$ $=0.90$ in the smooth phase, the critical value $C=1.00$ and $C$ $=2.00$ in the rough phase. Two system sizes $N=2048$ and $N$ $=4096$ are represented to better appreciate the deviations from power-law behavior, indicated with a dashed line, for the values outside the critical region. The curves correspond to an average over 500 realizations. 


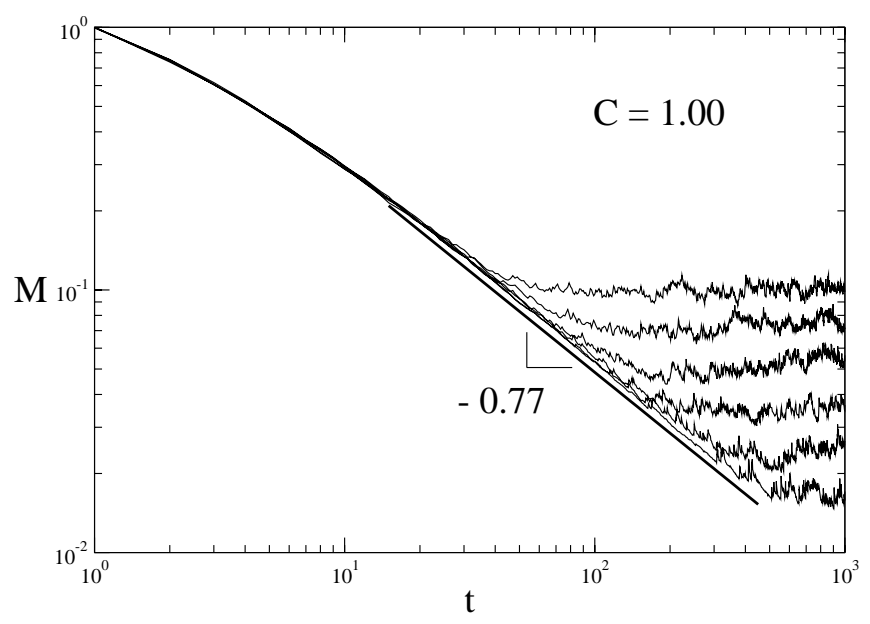

FIG. 7. Order parameter dynamics for six increasing values of the system size $N$, from top to bottom, $2^{9}-2^{14}$. A power-law behavior $M \sim t^{-0.77}$ is observed in the transient regime. Results correspond to averages over 500 realizations.

a power-law behavior is observed, while deviations occur for $C \neq C_{t h}$. The fit to a straight line in a log-log plot, as shown Fig. 7, leads to a determination of the ratio $\beta / \nu_{t}=0.77$ \pm 0.05 . One can write the dynamic scaling ansatz

$$
M(t, N, \epsilon)=N^{-\beta / \nu} \Phi\left(\epsilon t^{1 / \nu}, t / N^{z}\right)
$$

for the order parameter, which at the critical point $\epsilon=0$ reads

$$
M(t, N, \epsilon=0)=N^{-\beta / \nu} f\left(t / N^{z}\right),
$$

where the scaling function $f(u) \sim$ const for $u \gg 1$ and $f(u)$ $\sim u^{-\beta / \nu_{t}}$ for $u \ll 1$. We can then use the values of the exponents just obtained to collapse our data as shown in Fig. 8 with exponents $\nu_{t} \sim 1.43$ and $z \sim 0.57$.

Nonequilibrium phase transitions have been mostly related to the universality class of directed percolation (DP), with very few exceptions [13]. In particular, there are many

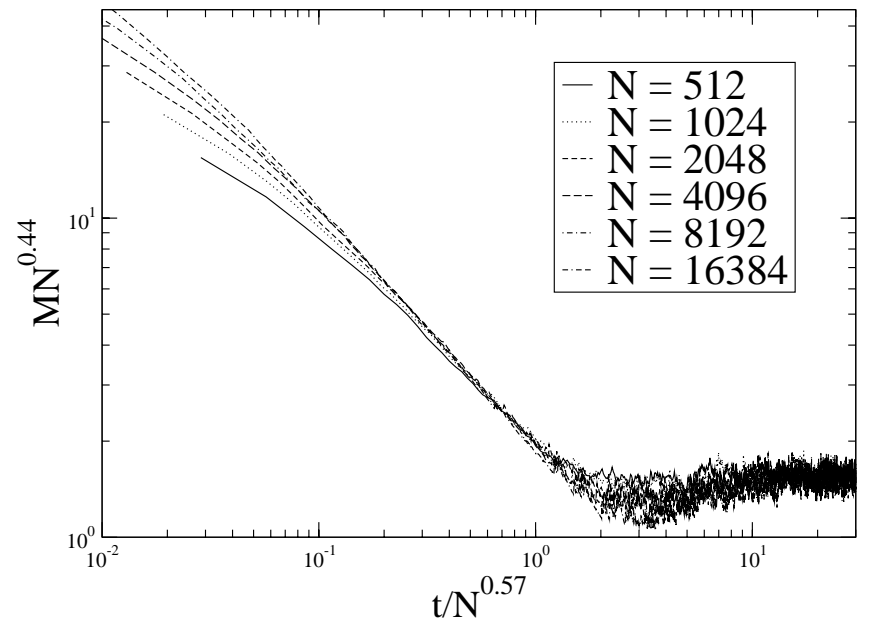

FIG. 8. Dynamical data collapse of the order parameter at the critical point, as given by Eq. (7). The exponents used correspond to the ones obtained above, $\beta / \nu \sim 0.44$, and $z \sim 0.57$. examples of roughening transitions far from equilibrium that have been linked to DP, examples include polynuclear growth models [18], solid-on-solid models with evaporation at the edges of terraces [16], and the fungal growth model [17]. In all these systems, the DP process emerges at a particular reference height of the interface. In this case, the critical exponents characterizing the roughening transition can be obtained from those of DP, which in $1+1$ dimension are given by $\nu=\nu_{\perp}^{D P}=1.10$ for the correlation length exponent, $\nu_{t}=\nu_{\|}^{D P}=1.73$ for the time correlation exponent, and $z$ $=z^{D P}=1.58$ the dynamic exponent [13]. Our results clearly suggest that the roughening transition occurring in the innovation propagation model does not belong to the DP class.

The relation of many nonequilibrium critical models to DP has led to the proposal of the conjecture due to Janssen and Grassberger $[19,20]$, which states that a model belongs to DP under the following assumptions [13]:

(1) The model displays a continuous phase transition from a fluctuating active phase into a unique absorbing state.

(2) The transition is characterized by a positive onecomponent order parameter.

(3) The dynamic rules involve only short-range interactions.

(4) Finally, the system has no special attributes like additional symmetries or quenched randomness.

Any model satisfying all above four conditions has been found to belong to DP universality class, with no exception to date. However, it is known that at least some of the above DP conditions can be relaxed. In fact, there are a few examples of systems that, despite exhibiting no absorbing states [16-18] or having quenched disorder [21,22] also display nonequilibrium phase transitions that belong to the DP universality class. Our model does not have absorbing states, since in both the rough and the flat phase the interface keeps fluctuating. Also and perhaps most importantly, interaction is not short ranged, because of the avalanches of activity that give rise to nonlocal effects with finite probability. Their influence on the dynamics is reflected by the extremely low value of the dynamic exponent, $z=0.57<2$, signature of a highly super diffusive behavior. It appears that this nonlocal interaction mechanism is responsible for the deviation of the DP critical behavior.

We believe that the transition takes place exactly at $C$ $=1.0$. This is directly related to the dynamical evolution rules of the model. We have defined the external driving by choosing a random number from a uniform distribution in $[0,1]$. As a consequence, for $C<1.0$, a random update on any site can generate an avalanche. On the other hand, for $C>1.0$, only a small fraction of sites will be able to generate an avalanche with a single update. In order to quantify this effect we have studied the fraction of sites which can generate an avalanche with a single update. A site $i$ with this property will satisfy

$$
h_{i}-h_{i \pm 1}+1>C \text {. }
$$

In Fig. 9 we present the fraction of sites $f$ which are able to generate an avalanche as a function of $\mathrm{C}$. The figure clearly 


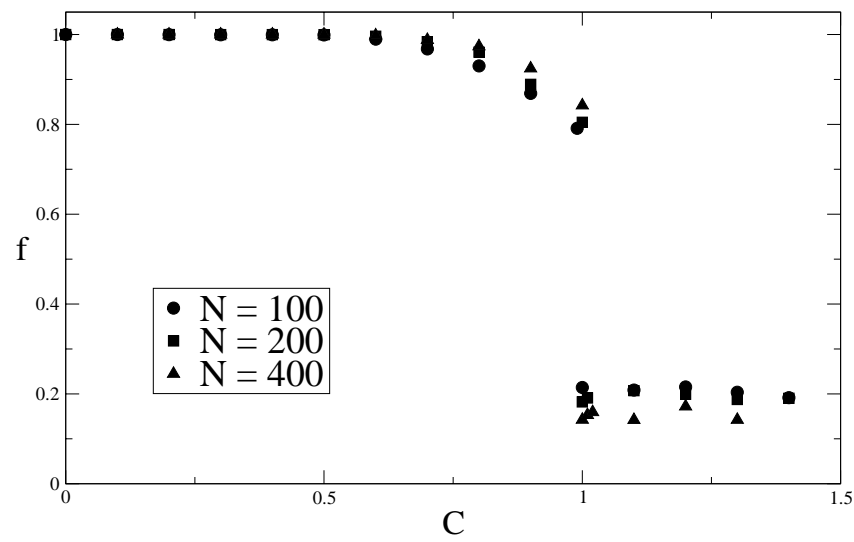

FIG. 9. Fraction of sites $f$ that can generate an avalanche with a single update as a function of $C$ for three different system sizes, $N=100, N=200$, and $N=400$. Curves are averaged over 1000 realizations.

shows that this fraction remains close to 1 for $C<1.0$ and drops abruptly to a small value for $C>1.0$.

\section{CONCLUSIONS}

In this work we have studied a simple model of innovation propagation dynamics in an economic system as a sur- face growth model. This has allowed us to characterize different morphological phases and also to analyze the dynamical behavior of the model as a kinetic roughening process. We have characterized a roughening transition and determined its critical exponents by finite-size scaling techniques. The values of the exponents do not coincide with known universality classes. We believe that the avalanche driven dynamics with its long-range effects is the reason why this model does not belong to the DP universality class. We have also presented a possible mechanism for the transition occurring exactly at $C_{t h}=1$.

\section{ACKNOWLEDGMENTS}

We would like to thank X. Guardiola, J.J. Ramasco, M.A. Rodríguez, A. Arenas, C.J. Pérez, and F. Vega-Redondo for fruitful discussions at the earliest stages of this work. M. Llas, P.M. Gleiser, and A. Díaz-Guilera acknowledge financial support from MCYT, Grant No. BFM2000-0626, and also from European Commission, Fet Open Project COSIN IST-2001-33555. P.M.G. acknowledges financial support from Fundación Antorchas. J.M.L. acknowledges support from the Ministerio de Ciencia y Tecnología (Spain) and FEDER under Project BFM2000-0628-C03-02.
[1] R. Albert and A.-L. Barabási, Rev. Mod. Phys. 74, 47 (2002).

[2] M.E.J. Newman, SIAM Rev. 45, 167 (2003).

[3] R.N. Mantegna and H.E. Stanley, An introduction to Econophysics: Correlations and Complexity in Finance (Cambridge University Press, Cambridge, 1999).

[4] J.P. Bouchaud and M. Potters, Theory of Financial Risk (Cambridge University Press, Cambridge, 2000).

[5] A. Arenas, A. Díaz-Guilera, C.J. Pérez, and F. Vega-Redondo, Phys. Rev. E 61, 3466 (2000).

[6] A. Arenas, A. Díaz-Guilera, C.J. Pérez, and F. Vega-Redondo, J. Econ. Dyn. Control 26, 2115 (2002).

[7] C. Castellano, M. Marsili, and A. Vespignani, Phys. Rev. Lett. 85, 3536 (2000).

[8] K. Klemm, V.M. Eguiluz, R. Toral, and M. San Miguel, Phys. Rev. E 67, 026120 (2003).

[9] X. Guardiola, A. Díaz-Guilera, C.J. Pérez, A. Arenas, and M. Llas, Phys. Rev. E 66, 026121 (2002).

[10] The agent-based approach used in the current work should be distinguished from models in which technological change is considered in a complex adaptive landscape. This is the case in W. Ebeling, Karmeshu, and A. Scharnhorst, in Econophysics: an Emerging Science, edited by J. Kertész and I. Kondor (Kluwer Scientific, Dordrecht, 1999), p. 293; Adv.
Complex Syst. 4, 71 (2001).

[11] X. Guardiola, Ph.D. thesis, Universitat de Barcelona, Spain, 2001 (unpublished).

[12] M. Llas, P.M. Gleiser, A. Díaz-Guilera, and C.J. Pérez, Physica A 326, 566 (2003).

[13] H. Hinrichsen, Adv. Phys. 49, 815 (2000).

[14] H.J. Jensen, Self-organized Criticality: Emergent complex Behavior in Physical and Biological Systems (Cambridge University Press, Cambridge, 1998).

[15] A.-L. Barabási and H.E. Stanley, Fractal Concepts in Surface Growth (Cambridge University Press, Cambridge, 1995).

[16] U. Alon, M.R. Evans, H. Hinrichsen, and D. Mukamel, Phys. Rev. E 57, 4997 (1998).

[17] J.M. López and H.J. Jensen, Phys. Rev. Lett. 81, 1734 (1998).

[18] J. Kertész and D.E. Wolf, Phys. Rev. Lett. 62, 2571 (1989).

[19] H.K. Janssen, Z. Phys. B: Condens. Matter 42, 151 (1981).

[20] P. Grassberger, Z. Phys. B: Condens. Matter 47, 365 (1982).

[21] L.-H. Tang and H. Leschhorn, Phys. Rev. A 45, R8309 (1992).

[22] S.V. Buldyrev et al., Phys. Rev. A 45, R8313 (1992).

[23] This election of the noise distribution is the only difference with model in Ref. [9]. The main properties of the model, among them the roughening transition, are robust under this change. 